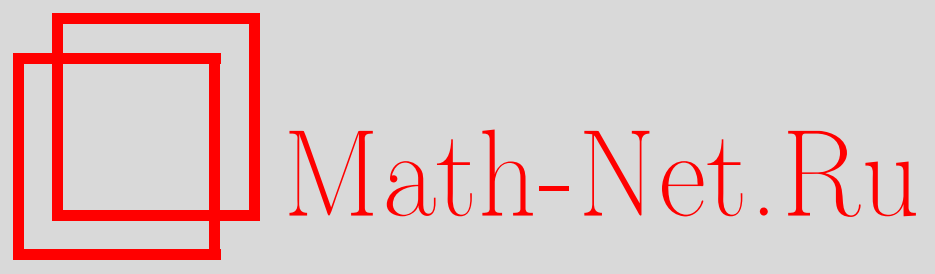

И. Л. Коган, Построение операторного исчисления Микусинского на основе алгебры свёртки обобщённых функций. Основные положения, Вестн. Сам. гос. техн. ун-та. Сер. Физ.-мат. науки, 2012, выпуск 2()$, 44-52$

DOI: https://doi.org/10.14498/vsgtu1013

Использование Общероссийского математического портала Math-Net.Ru подразумевает, что вы прочитали и согласны с пользовательским соглашением

http: //www . mathnet.ru/rus/agreement

Параметры загрузки:

IP : 3.85 .5 .30

26 апреля 2023 г., $16: 36: 26$

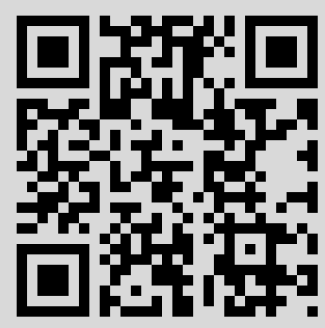


Вестн. Сам. гос. техн. ун-та. Сер. Физ.-мат. науки. 2012. № 2 (27). С. 44-52

\title{
Математический анализ
}

\author{
УДК 517.982.45
}

\section{ПОСТРОЕНИЕ ОПЕРАТОРНОГО ИСЧИСЛЕНИЯ МИКУСИНСКОГО НА ОСНОВЕ АЛГЕБРЫ СВЁРТКИ ОБОБЩЁННЫХ ФУНКЦИЙ. ОСНОВНЫЕ ПОЛОЖЕНИЯ}

\author{
И. Л. Коган \\ Российский государственный аграрный университет-MCХА им. К. А. Тимирязева, \\ 127550, Россия, Москва, Тимирязевская ул., 49. \\ E-mail: ik_@list.ru
}

\begin{abstract}
Даются основные положения операторного исчисления, основанного на использовании алгебры свёртки обобщённых функиий $D_{+}^{\prime}$ и $D_{-}^{\prime}$ и позволяющего распространить этот метод на область отрицательных значений аргумента. Приводится связь между предложенным подходом и классическим операционным исчислением, построенным на преобразовании Лапласа.
\end{abstract}

Ключевые слова: исчисление Микусинского, пространство обобщённых функций, свёртка обобщённых функиий, алгебра свёртки, преобразование Лапласа.

Введение. Классическое операционное исчисление, построенное на преобразовании Лапласа, ограничено функциями, для которых это преобразование применимо. Обойти ограничения позволяет оригинальный метод Микусинского [1], фактически использующий подход фундаментального решения уравнений. Особенностью метода является введение кольца операторов, причем под операторами понимаются числовые операторы, функции, заданные на положительной полуоси, и собственно операторы. В этом кольце вводятся линейные операции сложения и умножения на числа, а также свертка функций, рассматриваемая как операция умножения. Важнейшая черта метода использование обобщённых функций. Так, единичным числовым оператором по отношению к операции умножения является дельта-функция Дирака, а оператором интегрирования - единичная функция Хевисайда. Между операторным исчислением Микусинского и операционным исчислением на основании преобразования Лапласа существует формальное сходство: изображения функций по Лапласу совпадают с соответствующими изображениями по Микусинскому. Поэтому целесообразно дальнейшее развитие этого метода. Поставленной задачей является построение операторного исчисления Микусинского на более современной основе - алгебре свёртки обобщённых функций $[2,3]$, в которой роль операции умножения выполняет уже свёртка обобщённых функций. Поскольку свёрточную алгебру можно определить для обобщённых функций с носителями, лежащими отдельно на положительной и на отрицательной полуоси, то можно распространить метод на область отрицательных значений аргумента [4]. При этом удобно, учитывая связь с

Иосиф Леонидович Коган, старший преподаватель, каф. высшей математики. 
преобразованием Лапласа, определять изображения как функции комплексного переменного.

1. Базисные положения [1-3, 5]. Свёрткой двух функций $f(t)$ и $g(t)$, где $t \in \mathbb{R}$, в классическом анализе называется интеграл

$$
f * g=\int_{-\infty}^{\infty} f(\tau) g(t-\tau) d \tau=\int_{-\infty}^{\infty} g(\tau) f(t-\tau) d \tau
$$

Если $f$ и $g$ - две локально интегрируемые (суммируемые) функции и, кроме того, эти функции имеют ограниченные с одной и той же стороны носители, то свёртка всегда будет существовать и также являться локально интегрируемой функцией. При этих условиях операция свёртки ассоциативна, дистрибутивна относительно сложения и, по определению, коммутативна. Выделим случаи свёртки функций с носителями, лежащими на луче $[0 ; \infty)$ или $t \in \mathbb{R}_{+}$ и на луче $(-\infty ; 0]$ или $t \in \mathbb{R}_{-}$. Разбивая область интегрирования в исходной формуле (1), легко получить выражения для свёртки таких функций:

$$
\begin{gathered}
f * g=\int_{0}^{t} f(\tau) g(t-\tau) d \tau \quad\left(\operatorname{supp} f, g \in \mathbb{R}_{+}\right) \\
f * g=-\int_{0}^{t} f(\tau) g(t-\tau) d \tau \quad\left(\operatorname{supp} f, g \in \mathbb{R}_{-}\right) .
\end{gathered}
$$

Если свёртка $f * g$ существует, то для ее носителя имеет место включение

$$
\operatorname{supp}(f * g) \subset \overline{\operatorname{supp} f \cup \operatorname{supp} g} .
$$

Для свёртки функций справедлива теорема Титчмариа: если функции $f(t)$ и $g(t)$ непрерывны в области определения свёртки и $f * g=0$, то в этой области, по крайней мере, одна из функций тождественно равна нулю. Следствием теоремы является единственность представления свёртки двух непрерывных функиий.

Перейдём к свёртке обобщённых функций. Укажем основные используемые пространства. Пространство $D$ - линейное пространство бесконечно дифференцируемых (вообще комплекснозначных) финитных функций на $\mathbb{R}\left(\mathbb{R}^{n}\right)$. Пространство $D^{\prime}$ - линейное пространство обобщённых функций, заданных на $D$. Пространства $D_{+}^{\prime} \subset D^{\prime}$ и $D_{-}^{\prime} \subset D^{\prime}$ - пространства обобщённых функций с носителями, принадлежащими лучам $\mathbb{R}_{+}$и $\mathbb{R}_{-}$. Свёрткой двух обобщённых функций $f * g$ называется функционал

$$
f * g=(f * g, \varphi)=(f(x) \times g(y), \varphi(x+y)),
$$

где $\varphi \in D$, a $f(x) \times g(y)$ - прямое произведение функций. Равенство (4) определяет взаимно однозначное соответствие между свёрткой функций в классическом понимании и свёрткой обобщённых функций. Поэтому функцию, выраженную формулами (1) или (2), будем отождествлять с регулярным функционалом, заданным формулой (4), и наоборот. Кроме того, под равенством $f_{1} * f_{2}=g$ будем понимать как равенство функционалов, заданных в определённом пространстве, так и равенство обычных функций. Формула (4) всегда справедлива, если хотя бы один из функционалов $f$ и $g$ имеет ограниченный носитель, а также когда носители обоих функционалов одновременно 
лежат на луче $\mathbb{R}_{+}$или $\mathbb{R}_{-}$. Для носителя свёртки обобщённых функций верно соотношение (3). Аналогично определяется свёртка нескольких обобщённых функций, например, трёх обобщённых функций

$$
(f * g * w, \varphi)=(f(x) \times g(y) \times w(z), \varphi(x+y+z)) .
$$

Пространства $D_{+}^{\prime}$ и $D_{-}^{\prime}$ с операцией умножения - свёртка обобщённых функций образуют одноименные коммутативные алгебры с единицей $-\delta(t)$ (дельта-функцией Дирака). Элемент $a \in D_{+}^{\prime}\left(D_{-}^{\prime}\right)$ имеет обратный элемент $a^{-1}$ в этом же пространстве, если

$$
a * a^{-1}=a^{-1} * a=\delta .
$$

Обратный элемент единственен, поэтому уравнение в свёртках относительно $x(t): a * x=f$ имеет единственное решение $x=a^{-1} * f$. Элемент $a^{-1}$ называют элементарным, или фундаменталъным решением этого уравнения, т. е. решением, когда правая часть $f(t)=\delta(t)$. Отметим, что если $a-$ функция в классическом понимании, то $a^{-1}-$ сингулярная обобщённая функция, или оператор.

Для дифференцирования свёртки справедливо правило

$$
D^{n}(f * g)=D^{n} f * g=f * D^{n} g,
$$

где $D^{n}$ - оператор дифференцирования $n$-ного порядка в смысле теории обобщённых функций (в общем случае $n$ мультииндекс), которое распространяется для свёртки трёх и более обобщённых функций. Оператор дифференцирования можно выразить в виде свёртки

$$
D f=\delta^{\prime} * f ; \quad D^{n} f=\delta^{(n)} * f=\left(\delta^{\prime}\right)^{n} * f,
$$

где $\left(\delta^{\prime}\right)^{n}$ - свёрточный оператор дифференцирования $n$-ного порядка, или, в более общей форме,

$$
D^{n} f(t-h)=\left(\delta^{\prime}(t-h)\right)^{n} * f(t)
$$

Линейное дифференциальное уравнение с постоянными коэффициентами в алгебрах $D_{+}^{\prime}$ и $D_{-}^{\prime}$ в случае задания нулевых начальных условий эквивалентно свёрточным уравнениям

$$
L\left(\delta^{\prime}\right) * x(t)=\vartheta(t) f(t), \quad t \in \mathbb{R}_{+} ; \quad L\left(\delta^{\prime}\right) * x(t)=\vartheta(-t) f(t), \quad t \in \mathbb{R}_{-} .
$$

Здесь $\vartheta(t)$ - единичная функция Хевисайда, а $L\left(\delta^{\prime}\right)$ - линейный свёрточный оператор дифференцирования с постоянными коэффициентами:

$$
L\left(\delta^{\prime}\right)=\left(\delta^{\prime}\right)^{n}+a_{1}\left(\delta^{\prime}\right)^{n-1}+a_{2}\left(\delta^{\prime}\right)^{n-2}+\cdots+a_{n} \delta=\sum_{i=0}^{n} a_{n-i}\left(\delta^{\prime}\right)^{i}, \quad a_{0}=1 .
$$

Приведём теорему Лорана Швариа [2]. Оператор $L\left(\delta^{\prime}\right)$ всегда обратим в алгебре $D_{+}^{\prime}\left(D_{-}^{\prime}\right)$, т. е. существует фундаментальное решение для уравнений (7), имеющее вид

$$
\left(L\left(\delta^{\prime}\right)\right)^{-1}=\vartheta(t) x_{0}(t), \quad t \in \mathbb{R}_{+} ; \quad\left(L\left(\delta^{\prime}\right)\right)^{-1}=-\vartheta(-t) x_{0}(t), \quad t \in \mathbb{R}_{-},
$$


где $x_{0}(t)$ - решение однородного дифференциального уравнения, отвечающее начальным условиям

$$
x_{0}(0)=x_{0}^{\prime}(0)=\cdots=x_{0}^{(n-2)}(0)=0 ; \quad x_{0}^{(n-1)}=1 .
$$

Решение для произвольной правой части уравнений (7), независимо от рассматриваемого пространства:

$$
x(t)=\left(L\left(\delta^{\prime}\right)\right)^{-1} * f(t)=\int_{0}^{t} x_{0}(t-\tau) f(\tau) d \tau .
$$

Для ненулевых начальных условий

$$
x(0)=x_{0}, x^{\prime}(0)=x_{1}, \ldots, x^{(n-1)}(0)=x_{n-1}
$$

решение примет вид

$$
x(t)=\int_{0}^{t} x_{0}(t-\tau) f(\tau) d \tau+\sum_{k=0}^{n-1} e_{k} x_{0}^{(k)}(t)
$$

где $e_{k}=x_{n-1-k}+a_{1} x_{n-2-k}+\cdots+a_{n-k-1} x_{0}$. Сумма, входящая в правую часть формулы (12), является решением однородного дифференциального уравнения, отвечающим начальным условиям (11).

Рассмотрим некоторые соотношения для пространства $D_{+}^{\prime}$, используемые в дальнейшем. На основании теоремы Шварца, формул (9), (10) легко получить фундаментальные решения для простейших дифференциальных операторов, например,

$$
\left(\delta^{\prime}-\lambda \delta\right)^{-1}=\vartheta(t) e^{\lambda t} ; \quad\left(\delta^{\prime \prime}-\omega^{2} \delta\right)^{-1}=\vartheta(t) \sin \omega t / \omega .
$$

Оператор интегрирования можно представить в виде свёртки

$$
\vartheta(t) * f(t)=\int_{0}^{t} f(\tau) d \tau
$$

Свёрточные операторы дифференцирования (6) и интегрирования (14) являются обратными по отношению друг к другу, т. к. их свертка равна дельтафункции. Действительно, учитывая, что $(\vartheta(t))^{\prime}=\delta(t)$, по свойству дифференцирования свёртки (5) имеем $\vartheta(t) * \delta^{\prime}=(\vartheta(t))^{\prime} * \delta(t)=\delta(t)$.

Выражение $[\vartheta(t)]^{n}$, где $n \in \mathbb{N}$, представляет $n$-кратный свёрточный оператор интегрирования. Согласно известной формуле Коши для $n$-кратного интеграла по интервалу от 0 до $t$ имеем

$$
[\vartheta(t)]^{n} * f(t)=\frac{1}{(n-1) !} \int_{0}^{t} f(t-\tau) \tau^{n-1} d \tau=\vartheta(t) t^{n-1} /(n-1) ! * f(t) .
$$

Отсюда

$$
*[\vartheta(t)]^{n}=* \vartheta(t) t^{n-1} /(n-1) ! .
$$

Символ «*», стоящий перед выражениями в правой и левой частях последней формулы, указывает, что это равенство свёрточных операторов. Обобщением 
этого оператора является свёрточный оператор дробного интегрирования для любого вещественного $\lambda>0$, который можно представить в виде

$$
*[\vartheta(t)]^{\lambda}=* \vartheta(t) t^{\lambda-1} / \Gamma(\lambda),
$$

или, введя числовой параметр $\alpha[1$, с. 110],

$$
*\left[\vartheta^{-1}(t)-\alpha\right]^{-\lambda}=* \vartheta(t) t^{\lambda-1} e^{\alpha t} / \Gamma(\lambda) .
$$

Отметим, что можно также ввести операторы дробного дифференцирования при $\lambda<0\left[3\right.$, с. 88], [5, с. 133], используя $*[\vartheta(t)]^{-n}=*\left[\delta^{\prime}(t)\right]^{n}$.

Теперь вернемся к пространству $D_{-}^{\prime}$. Здесь также можно ввести свёрточный оператор интегрирования, который является обратным по отношению к оператору дифференцирования (6):

$$
(-\vartheta(-t)) * f(t)=\int_{0}^{t} f(\tau) d \tau .
$$

В самом деле,

$$
(-\vartheta(-t)) * \delta^{\prime}=(-\vartheta(-t))^{\prime} * \delta(t)=\delta(t) .
$$

Для целой степени этого оператора справедлива формула, аналогичная (15), T. e.

$$
*[-\vartheta(-t)]^{n}=*\left(-\vartheta(-t) t^{n-1} /(n-1) !\right) .
$$

Таким образом, можно сделать следующий вывод: формулы для фундаментальных решений простейших дифференциальных операторов (теорема Шварца) и целочисленных операторов интегрирования в пространстве $D_{-}^{\prime}$ отличаются от соответствующих формул для пространства $D_{+}^{\prime}$ только заменой множителя $\vartheta(t)$ на множитель $(-\vartheta(-t))$.

2. Основы построения теории. Исходные уравнения, функции и операторы будем рассматривать отдельно в пространствах $D_{+}^{\prime}$ и $D_{-}^{\prime}$. Введём следующие определения.

Функцией-оригиналом в классическом понимании называется любая кусочно-непрерывная, локально интегрируемая комплекснозначная функция $f(t)$ действительного аргумента $t, \operatorname{supp} f \in \mathbb{R}_{+}$или $\mathbb{R}_{-}$, имеющая на каждом конечном интервале не более чем конечное число точек разрыва. Обозначим пространства таких функций, соответственно, как $K_{+}$и $K_{-}$. Производные дельта-функции Дирака - $\delta^{(n)}(t)$, где $n=0,1,2, \ldots$, также являющиеся оригиналами, можно рассматривать как импульсные функиии $n$-ного порядка [6, с. 494] и как операторы.

Изображением функиии или оператора $f(t)$ является функция комплексного переменного $F(p)(p=s+i \omega)$, аналитическое выражение которой определяется соотношением

$$
f(t) *\left(F\left(\delta^{\prime}\right)\right)^{-1}=\delta(t),
$$

в котором аргумент изображения $p$ заменён на $\delta^{\prime}$. Иначе, $f(t) \leftrightarrow F(p)$ или $F(f(t))=F(p)$, где $p$ - комплексная переменная, если имеет место равенство элементов свёрточной алгебры

$$
* f(t)=* F\left(\delta^{\prime}\right) .
$$


При таком задании свёрточного преобразования $F(f(t))$ сходимость изображений сводится к сходимости функций комплексного переменного, и можно установить связь предложенного подхода с операционным исчислением на основании преобразования Лапласа. Из определения (18) или (19) сразу следует теорема единственности.

Теорема 1. Между оригиналом $f(t)$ и изображением $F(p)$ существует взаимнооднозначное соответствие.

Д о ка з а т е ль с т в о. Действительно, эта теорема вытекает из единственности для обратного элемента в свёрточной алгебре, в данном случае $D_{+}^{\prime}$ $\left(D_{-}^{\prime}\right)$.

Введём изображения простейших свёрточных операторов (см. таблицу). Условимся считать, если не оговорено особо, что рассматривается пространство $D_{+}^{\prime}\left(f(t) \in K_{+}\right)$. Поэтому множитель $\vartheta(t)$ перед выражением оригинала будем опускать. Для пространства $D_{-}^{\prime}\left(f(t) \in K_{-}\right)$дополнительный множитель $\vartheta(-t)$ перед выражением оригинала также не будем вводить. Формулы соответствия между функцией-оригиналом и её изображением приводятся только для пространства $D_{+}^{\prime}$. В случае пространства $D_{-}^{\prime}$ эти формулы, если, конечно, функции определены при $t \in \mathbb{R}_{-}$, отличаются от соответствующих формул для пространства $D_{+}^{\prime}$ только знаком «минус» перед выражением функции. Например, для единичной функции $\vartheta(t)$ в $D_{+}^{\prime}$ формула соответствия $1 \leftrightarrow 1 / p$, а для единичной функции в $\vartheta(-t) D_{-}^{\prime}$ аналогичная формула $(-1) \leftrightarrow 1 / p$. Отметим, что изображение дельта-функции и её производных одинаково в обоих пространствах.

\begin{tabular}{l|c|c}
\hline \multicolumn{1}{c|}{ Оператор (функция), операция } & \multirow{2}{*}{ Изображение } \\
\hline Назание & Обозначение & \\
Операция «свёртка функций» & $*$ & $\cdot$ \\
Единичный оператор & $\delta(t)$ & 1 \\
Оператор дифференцирования & $\delta^{\prime}(t)$ & $p$ \\
Оператор интегрирования & $-\vartheta(-t),\left(D_{-}^{\prime}\right)$ & $1 / p$ \\
(единичная функция) & $\vartheta(t),\left(D_{+}^{\prime}\right)$ & \\
\hline
\end{tabular}

Далее из определения свёрточного преобразования (18) или (19), формул для степени свёрточных операторов интегрирования (15), (16) и (17) и таблицы непосредственно следуют формулы

$$
\begin{aligned}
& t^{n} \leftrightarrow n ! / p^{n+1}, \quad n=0,1,2, \ldots \\
& t^{\lambda} \leftrightarrow \Gamma(\lambda+1) / p^{\lambda+1}, \quad \lambda>-1 ; \\
& t^{\lambda} e^{\alpha t} \leftrightarrow \Gamma(\lambda+1) /(p-\alpha)^{\lambda+1}, \quad \lambda>-1 .
\end{aligned}
$$

Теорема 2. Свёрточное преобразование (18) или (19) линейно. Пусть $f_{i}(t) \leftrightarrow F_{i}(p)$, где $i=1,2, \ldots, n$, тогда для любых (комплексных) постоянных $c_{i}$

$$
\sum_{i=1}^{n} c_{i} f_{i}(t) \leftrightarrow \sum_{i=1}^{n} c_{i} F_{i}(p) .
$$


Доказ а тель ст в о. Линейная комбинация свёрточных равенств, записанных в символическом виде, действительно является свёрточным равенством, так как $D_{+}^{\prime}\left(D_{-}^{\prime}\right)$ - линейное пространство. $\square$

ТЕорема 3. Если оригинал $f(t)$ является фундаментальным решением линейного дифберенциального уравнения с постоянными коэфбициентами (7), отвечающим нулевым начальным условиям, то его изображсением будет функиия $F(p)$, обратная свёрточному оператору дифберениирования для этого уравнения, записанная в символическом виде

$$
f(t) \leftrightarrow(L(p))^{-1}=F(p),
$$

где $L(p)$ определяется формулой (8), в которой аргумент $\delta^{\prime}$ заменен на р. При этом выполняется предельное соотношение

$$
\lim _{s \rightarrow \infty(-\infty)} F(p)=0, \quad s=\operatorname{Re} p
$$

Доказ ат ельств о. Действительно, по условию теоремы $L\left(\delta^{\prime}\right) * f(t)=$ $=\delta(t)$. Отсюда $* f(t)=*\left(L\left(\delta^{\prime}\right)\right)^{-1}$ и на основании определения свёрточного преобразования (18) или (19) следует (20). Формальное соотношение (21) вытекает из вида выражения $F(p)$.

Теоремы 2 и 3 (формулы $(9),(10))$ позволяют очевидным образом строить таблицу оригиналов и их изображений. В частности, из (13) сразу следуют формулы

$$
e^{\lambda t} \leftrightarrow 1 /(p-\lambda), \quad \sin \omega t \leftrightarrow \omega /\left(p^{2}+\omega^{2}\right)
$$

ТЕОРЕМА 4 (ТЕОРЕМА УМНОЖЕНИЯ ИЗОБРАЖЕНИЙ Э. БОРЕЛЯ). Пустъ

$$
f_{i}(t) \leftrightarrow F_{i}(p)
$$

где $i=1,2, \ldots, n$, тогда

$$
\left(f_{1}(t) * f_{2}(t) * \ldots * f_{n}(t)\right)=* \prod_{i=1}^{n} f_{i}(t) \leftrightarrow \prod_{i=1}^{n} F_{i}(p) .
$$

Доказ ательств о. Это соотношение следует из свойства ассоциативности алгебры свёртки и определения свёрточного преобразования (18) или (19).

ТЕОРЕМА 5 (ТЕОРЕМА ДИФФЕРЕНЦИРОВАНИЯ И ИНТЕГРИРОВАНИЯ ОРИГИНАЛА). Пусть $f(t) \leftrightarrow F(p)$, тогда

$$
D f \leftrightarrow p F(p), \quad D^{n} f \leftrightarrow p^{n} F(p), \quad \int_{0}^{t} f(\tau) d \tau \leftrightarrow F(p) / p .
$$

Дока з а т ель с т в о немедленно следует из определения свёрточных операторов (см. таблицу) и теоремы умножения (теорема 4):

$$
D f=\delta^{\prime} * f \leftrightarrow p F(p) ; \quad D^{n} f=\left(\delta^{\prime}\right)^{n} * f \leftrightarrow p^{n} F(p)
$$




$$
\begin{gathered}
\int_{0}^{t} f(\tau) d \tau=\vartheta(t) * f \leftrightarrow F(p) / p, \quad f(t) \in K_{+} ; \\
\int_{0}^{t} f(\tau) d \tau=-\vartheta(-t) * f \leftrightarrow F(p) / p, \quad f(t) \in K_{-} .
\end{gathered}
$$

ТЕОРЕМА 6 (ТЕОРЕМА ОБ ИЗОБРАЖЕНИИ КЛАССИЧЕСКИХ ПРОИЗВОДНЫХ). Формуль для изображений различны в пространствах $D_{+}^{\prime} u D_{-}^{\prime}$.

Пусть $f(t) \in C^{n}\left(\mathbb{R}_{+}\right) \subset K_{+}(n=1,2, \ldots)$ u $f(t) \leftrightarrow F(p)$, тогда изображение обычной производной $f_{c l}^{\prime}(t)$ от этой функиии в $D_{+}^{\prime}$ определяется из формулы

$$
f_{c l}^{\prime}(t) \leftrightarrow p F(p)-f(0), \quad t \in \mathbb{R}_{+} .
$$

Пусть $f(t) \in C^{n}\left(\mathbb{R}_{-}\right) \subset K_{-}(n=1,2, \ldots)$ u $f(t) \leftrightarrow F(p)$, тогдав в $D_{-}^{\prime}$

$$
f_{c l}^{/}(t) \leftrightarrow p F(p)+f(0), \quad t \in \mathbb{R}_{-} .
$$

Доказ ательств о. Для доказательства (22) представим $f(t)$ в виде $f(t)=\vartheta(t) f(t)$. Дифференцирование этого выражения в смысле теории обобщённых функций даёт $D f=f(0) \delta(t)+\vartheta(t) f_{c l}^{\prime}(t)$. Переходя к изображениям, согласно свойству линейности (теорема 2) и предыдущей теоремы, $p F(p)=$ $=f(0)+F\left(f_{c l}^{\prime}(t)\right)$, откуда следует $(22)$. Общая формула для изображения классической производной $n$-того порядка имеет вид

$$
f_{c l}^{(n)}(t) \leftrightarrow p^{n} F(p)-p^{n-1} f(0)-p^{n-2} f^{\prime}(0)-\ldots-f^{(n-1)}(0), \quad t \in \mathbb{R}_{+}
$$

Доказательство (23) аналогично доказательству (22) и основано на представлении $f(t)=\vartheta(-t) f(t)$. Общая формула для изображения классической производной $n$-ного порядка имеет вид

$$
f_{c l}^{(n)}(t) \leftrightarrow p^{n} F(p)+p^{n-1} f(0)+p^{n-2} f^{\prime}(0)+\ldots+f^{(n-1)}(0), \quad t \in \mathbb{R}_{-} . \square
$$

Введём пространство функций $M$, для которых применимо преобразование Лапласа. Очевидно, $M \subset K_{+}$. Имеет место утверждение: для любой бункиии $f(t) \in M$ свёрточное преобразование в пространстве $D_{+}^{\prime}$ совпадает с преобразованием Лапласа. Действительно, при выбранном подходе формулировки основных теорем и формул для пространства $D_{+}^{\prime}$ полностью идентичны соответствующим теоремам и формулам для операционного исчисления на основе преобразования Лапласа. Так, для обоих преобразований изображение единичного оператора в алгебре свёртки - $\delta(t)$ равно единице и справедлива теорема умножения изображений.

Таким образом, при реализации свёрточного преобразования в пространстве $D_{+}^{\prime}$ можно воспользоваться многочисленными таблицами преобразования Лапласа; в случае пространства $D_{-}^{\prime}$ формулы соответствия между функцией-оригиналом и её изображением отличаются от соответствующих формул для пространства $D_{+}^{\prime}$, только знаком «минус» перед выражением функции. 


\section{БИБЛИОГРАФИЧЕСКИЙ СПИСОК}

1. Mikusinski J. Operational calculus. New York: Pergamon Press, 1959. 495 pp.; pyccк. пер.: Микусинский Я. Операторное исчисление. М.: Иностр. лит., 1956. 366 с.

2. Schwartz L. Méthodes mathématiques pour les sciences physiques. Paris: Hermann, 1961. 39 рр.; русск. пер.: Швари Л. Математические методы для физических наук. М.: Мир, 1965. $412 \mathrm{c}$.

3. Владимиров B.C. Обобщённые функции в математической физике. М.: Наука, 1979. 319 c. [Vladimirov V.S. Generalized functions in mathematical physics. Moscow: Nauka, 1979. 319 pp.]

4. Коган И. Л. Метод интеграла Дюамеля для обыкновенных дифференциальных уравнений с постоянными коэффициентами с точки зрения теории обобщенных функций // Вестн. Сам. гос. техн. ун-та. Сер. Физ.-мат. науки, 2010. №1(20). С. 37-45. [Kogan I. L. Method of Duhamel integral for ordinary differential equations with constant coefficients in respect to the theory of distributions // Vestn. Samar. Gos. Tekhn. Univ. Ser. Fiz.-Mat. Nauki, 2010. no. 1(20). Pp. 37-45].

5. Гельфанд И. М., Шилов Г. Е. Обобщённые функции. Т. 1: Обобщённые функции и действия над ними. М.: Физматлит, 1959. 470 с.; англ. пер.: Gel'fand I. M., Shilov G.E. Generalized functions. Vol. 1: Properties and operations. New York: Academic Press, 1964. $423 \mathrm{pp}$.

6. Лаврентъев М.А., Шабат Б. В. Методы теории функций комплексного переменного. M.: Наука, 1987. 688 c. [Lavrent'ev M. A., Shabat B. V. Methods of the theory of functions in a complex variable. Moscow: Nauka, 1987. 688 pp.]

Поступила в редакцию 18/X/2011;

в окончательном варианте - 22/III/2012.

MSC: 44A40; 46T30

\section{CONSTRUCTION OF MIKUSINSKI OPERATIONAL CALCULUS BASED ON THE CONVOLUTION ALGEBRA OF DISTRIBUTIONS. BASIC PROVISIONS}

\section{L. Kogan}

Russian State Agrarian University - Moscow Agricultural Academy after K. A. Timiryazev, 49, Timiryazevskaya str., Moscow, 127550, Russia.

E-mail: ik_@list.ru

The main provisions of the operational calculus based on the convolution algebra of distributions $D_{+}^{\prime}$ and $D_{-}^{\prime}$ that extends this method to the negative values of the argument are given. The relation between the proposed method and the classical operational calculus built on the Laplace transform is provided.

Key words: calculus of Mikusinski, space of distributions, convolution of distributions, convolution algebra, Laplace transform.

Original article submitted 18/X/2011; revision submitted $22 / \mathrm{III} / 2012$.

Iosif L. Kogan, Senior Lecturer, Dept. of Higher Mathematics. 